\title{
Nota Científica \\ Qualidade fisiológica e sanitária de sementes de açoita-cavalo (Luehea divaricata)
}

\author{
Caciara Gonzatto Maciel ${ }^{1}$, Letiele Bruck De Souza², Marciéli Pitorini Bovolini', Daniele Lemos Brum¹, Marlove Fátima Brião Muniz¹, \\ Galileu Adeli Buriol² \\ ${ }^{1}$ Universidade Federal de Santa Maria, Departamento de Defesa Fitossanitária, Prédio 42, Campus Universitário, CEP 97105-900, \\ Santa Maria, RS, Brasil \\ ${ }^{2}$ Universidade Federal de Santa Maria, Programa de Pós-Graduação em Agrobiologia, Avenida Roraima, n 1000, Prédio 16, CEP 97105-900, Santa Maria, RS, Brasil
}

"Autor correspondente:
caciaragonzatto@gmail.com

Termos para indexação:

Germinação

Sementes florestais

Patologia de sementes

Index terms:

Germination

Forest seeds

Seed pathology

Histórico do artigo:

Recebido em 02/12/2012

Aprovado em 14/08/2013

Publicado em 30/09/2013

doi: 10.4336/2013.pfb.33.75.457
Resumo - Objetivou-se com esse trabalho caracterizar dois lotes de sementes de Luehea divaricata, quanto à sua qualidade fisiológica e sanitária. As sementes foram coletadas nos municípios de Santa Maria (SM) e Restinga Seca (RES) - região central do Rio Grande do Sul. Foi utilizado o substrato comercial Carolina Soil $\mathbb{R}$ para a caracterização fisiológica e o teste de transmissão de fungos via sementes. As variáveis determinadas foram índice de velocidade de germinação (IVG), emergência (\%), plântulas sintomáticas (\%) e sementes mortas (\%). Para a detecção dos fungos, foram utilizados os testes em BDA e substrato papel-filtro (PF). Para cada teste foram utilizadas quatro repetições de 25 sementes. Determinou-se ainda a sanidade dos frutos, através do blotter test. O IVG e o percentual de emergência variaram entre 6,7 e 8,7; 51 e 63\%, para os lotes RES e SM, respectivamente. Os fungos identificados associados às sementes, independente do método foram: Fusarium sp., Alternaria sp., Rhizoctonia sp., Aspergillus sp., Penicillium sp., Pestalotiopsis sp., Phoma sp. e Cladosporium sp. Destes, os gêneros Fusarium e Alternaria, podem estar associados aos sintomas observados nas plântulas no teste transmissão. Os fungos identificados presentes nos frutos foram: Fusarium sp., Aspergilus sp., Penicillium sp., Cladosporium sp. e Alternaria sp.

\section{Physiological and sanitary quality of seeds of açoita-cavalo (Luehea divaricata)}

\begin{abstract}
The objective of this study is to characterize two lots of Luehea divaricate seeds, as its physiological and sanitary quality. Seeds were collected in the municipalities of Santa Maria (SM) and Restinga Seca (RES), located inthe central region of Rio Grande do Sul State, Brazil. Germination tests and fungi transmission were conducted in commercial substrate Carolina Soil ${ }^{\circledR}$. The variables determined were germination indices (GI), emergency (\%), symptomatic seedlings (\%) and dead seeds (\%). The fungi were detected using PDA test and substrate filter paper (PF). For each test it was used four replicates of 25 seeds. It has been also determined the sanity of the fruits using blotter test. The GI and percentage of emergence varied between 6.7 and 8.7 and 51 and $63 \%$, for RES and SM lots, respectively. The fungi associated with the seeds, independent of the method were: Fusarium sp. Alternaria sp., Rhizoctonia sp. Aspergillus sp., Penicillium sp., Pestalotiopsis sp., Phoma sp. and Cladosporium sp. Fusarium and Alternaria, can be associated with symptoms in seedlings of the transmission test. The fungi presents in fruits were: Fusarium sp., Aspergilus sp., Penicillium sp., Cladosporium sp. and Alternaria sp.
\end{abstract}


Luehea divaricata Mart. é uma árvore pertencente à família Malvaceae, que apresenta madeira de boa qualidade, indicada para confecção de móveis e construção civil. Pela beleza de suas flores, recomendase o uso do açoita-cavalo em arborização de rodovias, praças e parques, além de ser indicada para recuperação de áreas de preservação permanente (Carvalho, 2008). De acordo com Sarmento \& Vilela (2010), essa espécie é melífera com propriedades medicinais, e com potencial para produção de celulose e móveis.

Existem estudos para determinação da qualidade sanitária de sementes de espécies florestais, porém, são poucos os trabalhos que concluem se esses fungos associados às sementes podem ser transmitidos para a plântula. Segundo Benetti et al. (2009), a baixa qualidade das mudas de certas espécies florestais nativas pode estar relacionada a problemas fitossanitários nas suas sementes, havendo assim necessidade de detecção para posterior controle desses patógenos. Maciel et al. (2012) identificaram os gêneros Penicillium, Aspergilus, Rhyzopus, Epicoccum, Thielaviopsis, Cladosporium, Fusarium, Pestalotia, Cladosporium, Phoma e Nigrospora associados a sementes de angicovermelho (Parapiptadenia rigida), sendo que Fusarium spp. foi patogênico às plântulas, causando apodrecimento dos cotilédones e má formação do sistema radicular; Souza et al. (2012) encontraram os gêneros Aspergillus spp., Curvularia sp., Penicillium sp., Pestalotia sp. e Fusarium spp. em sementes de ipê-rosa (Tabebuia impetiginosa), e, Aspergillus spp., Curvularia sp., Fusarium spp., Phoma sp. e Nigrospora sp. em sementes de ipê-amarelo (Tabebuia ochracea); Botelho et al. (2008) verificaram 16 gêneros fúngicos distintos associados a sementes de ipê-roxo (Tabebuia heptaphylla (Vell.)Tol.) e amarelo (Tabebuia chysotricha Mart. ex. DC. Stand1.), e dentre eles os mais frequentes transmitidos pelas sementes para as plântulas foram: Alternaria alternata, Fusarium spp., Aspergillus spp., Phoma sp. e Phomopsis sp.; Silva et al. (2011) encontraram os gêneros Fusarium, Aspergillus, Alternaria e Penicillium em sementes de angico-vermelho (Anadenanthera macrocarpa Benth.), jacarandá-da-Bahia (Dalbergia nigra (Vell.) Fr. All.ex.), cássia-do-Sultão (Senna siamea Lam), ipê-roxo (Tabebuia heptaphylla) e ipê-amarelo (Tabebuia chysotricha).

Relatos da incidência e influência dos patógenos associados a espécies florestais vêm aumentando, entretanto no caso do açoita-cavalo ainda são escassos. Bernardi-Wenzel et al. (2010) relataram a ocorrência de fungos endofíticos associados a folhas de Luehea divaricata coletadas em Maringa, PR, sendo eles os gêneros Alternaria, Cochliobolus, Diaporthe, Epicoccum, Guignardia, Phoma, e Phomopsis.

Este trabalho teve como objetivos: avaliar a sanidade das sementes de açoita-cavalo através dos métodos, blotter test e plaqueamento em meio BDA; avaliar a possível transmissão de fungos associados aos frutos para as sementes e destas para as plântulas; e determinar o potencial fisiológico das sementes através das variáveis índice de velocidade de emergência (IVG), emergência (\%) plântulas sintomáticas (\%) e sementes mortas (\%).

Os frutos foram coletados nas árvores no mês de dezembro de 2011, sendo de duas matrizes na área do Jardim Botânico nas dependências da Universidade Federal de Santa Maria, em Santa Maria, RS (2942'S e $\left.53^{\circ} 42^{\prime} \mathrm{W}\right)$; e de seis matrizes no município de Restinga

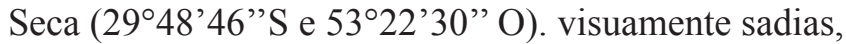
com copa bem distribuída e fuste reto, conforme descrito por Piña Rodrigues (2002).

Os frutos permaneceram por sete dias em temperatura ambiente $\left(25 \pm 2{ }^{\circ} \mathrm{C}\right)$ para secagem e posterior extração das sementes. Foram reservados 100 frutos de cada lote para a realização do teste de sanidade, com o objetivo de verificar se os fungos presentes nas sementes também estavam associados aos frutos.

Foram utilizadas 100 sementes, divididas em quatro repetições de 25 para cada lote. Utilizou-se o substrato Carolina Soil ${ }^{\circledR}$ autoclavado a $121{ }^{\circ} \mathrm{C}$ durante $60 \mathrm{~min}$, duas vezes consecutivas, com um intervalo de $24 \mathrm{~h}$ entre cada esterilização. $\mathrm{O}$ substrato foi acondicionado em caixas de plástico transparente (gerbox) onde foi realizada a semeadura, 25 sementes por caixa. O material permaneceu incubado na sala de germinação $\left(25 \pm 2^{\circ} \mathrm{C}\right)$ e fotoperíodo de $12 \mathrm{~h}$ com lâmpadas fluorescentes do tipo luz do dia, com irrigação manual sempre que necessário. As variáveis avaliadas foram: a) índice de velocidade de emergência: metodologia proposta por Maguire (1962); b) percentual de emergência: foram contabilizadas as plântulas normais aos 34 dias, quando estas tiveram sua emergência estabilizada. Adotou-se o critério plântula normal proposto por Brasil (2009); c) percentual de sementes mortas: compreende aquelas sementes que não apresentaram protrusão da radícula; d) plântulas anormais sintomáticas: correspondem às plântulas anormais que apresentaram sintomas da presença de fungos, sendo estas retiradas e incubadas em caixas de plástico transparente (gerbox) a $25 \pm 2{ }^{\circ} \mathrm{C}$ e fotoperíodo de $12 \mathrm{~h}$, onde permaneceram 
por cinco dias. Posteriormente, fez-se a identificação dos gêneros fúngicos associados aos sintomas, com auxilio de microscópio estereoscópico e óptico e bibliografia especializada (Barnett \& Hunter, 1999).

Para PF, foram utilizadas 100 sementes de cada lote, não desinfestadas, divididas em quatro repetições, com 25 sementes, distribuídas em caixas de plástico transparente (gerbox), previamente desinfestadas com solução de hipoclorito de sódio $1 \%$ e álcool $70 \%$, forradas com duas folhas de papel-filtro esterilizadas e umedecidas com água destilada esterilizada.

No caso do teste em meio BDA foram utilizadas 100 sementes de cada lote, divididas em quatro repetições de 25. A assepsia das sementes foi realizada com a imersão destas em álcool $70 \%$ por 30 segundos, depois em hipoclorito de sódio na concentração de $1 \%$ por 30 segundos e, em seguida, lavadas em dois banhos de água destilada esterilizada. Posteriormente, estas foram plaqueadas em meio de cultura BDA. A incubação para $\mathrm{PF}$ e BDA foi feita a uma temperatura de $22 \pm 3{ }^{\circ} \mathrm{C}$, e fotoperíodo de $12 \mathrm{~h}$, durante sete dias, quando ocorreu a avaliação e identificação dos fungos, com auxilio do microscópio estereoscópico e óptico e bibliografia especializada (Barnett \& Hunter, 1999).

Foram utilizados 100 frutos por lote, divididos em quatro repetições de 25. Devido à alta incidência de fungos saprófitas nos frutos, que são naturalmente pilosos, fezse uma assepsia de 15 minutos em hipoclorito de sódio $1 \%$, e posterior banho em água destilada esterilizada por um minuto. $\mathrm{O}$ material permaneceu secando em temperatura ambiente $\left(22 \pm 3{ }^{\circ} \mathrm{C}\right) \mathrm{e}$, posteriormente, foi distribuído em caixas de plástico transparente (gerbox) contendo duas folhas de papel-filtro umedecidas com água esterilizada, onde permaneceu por sete dias, para posterior identificação dos gêneros fúngicos presentes, como descrito para os testes de sanidade em PF e BDA.

A análise dos dados foi efetuada inicialmente pela verificação da normalidade com o teste Shapiro Wilk. Nos casos onde os dados não apresentaram uma distribuição normal, aplicou-se a transformação $\sqrt{x}+0,5$, onde $\mathrm{x}$ corresponde ao valor a ser transformado. Aplicouse a análise de variância e o teste de Tukey, ao nível de $5 \%$ de significância.

Os resultados quanto à caracterização fisiológica dos lotes estão apresentados na Tabela 1. Para o índice de velocidade de emergência os valores variaram entre 6,7 e 8,7, para os Lotes RES e SM, respectivamente.
Tabela 1. Índice de velocidade de emergência (IVG), emergência (\%), plântulas sintomáticas (\%) e sementes mortas (\%) de açoita-cavalo, coletadas nos municípios de Santa Maria (SM) e Restinga Seca (RES).

\begin{tabular}{ccccc}
\hline & IVG & $\begin{array}{c}\text { Emergência } \\
\mathbf{( \% )}\end{array}$ & $\begin{array}{c}\text { Plântulas } \\
\text { sintomáticas } \\
(\mathbf{\%})\end{array}$ & $\begin{array}{c}\text { Sementes } \\
\text { mortas } \\
\mathbf{( \% )}\end{array}$ \\
\hline $\begin{array}{c}\text { Lote } 1 \\
(\mathrm{SM})\end{array}$ & $8,7 \mathrm{a}$ & $63 \mathrm{a}^{*}$ & $0 \mathrm{a}$ & $37 \mathrm{a}$ \\
$\begin{array}{c}\text { Lote } 2 \\
\text { (RES) }\end{array}$ & $6,7 \mathrm{a}$ & $51 \mathrm{a}$ & $4 \mathrm{a}$ & $45 \mathrm{a}$ \\
\hline
\end{tabular}

* Médias seguidas pela mesma letra, na coluna, não diferem estatisticamente entre si pelo teste de Tukey ao nível de 5\% de significância.

A emergência das sementes de Luehea divaricata variou entre $51 \%$ e $63 \%$, para os Lotes SM e RES, respectivamente, e as contagens foram até 34 dias após a semeadura (Figura 1C). Utilizando substrato vermiculita, Borges et al. (2007) encontram valores de emergência que variam de $30,4 \%$ a $51 \%$ para sementes de açoitacavalo coletadas em Minas Gerais.

No trabalho realizado por Silva (2004) o percentual de emergência de sementes desta mesma espécie, oriundas da região metropolitana de Porto Alegre, RS, atingiu $75 \%$, tendo como substrato areia. Essas variações nos valores de emergência de uma região para outra, ou até mesmo dentro de uma mesma região, podem estar associadas a fatores como condições de solo, umidade e insolação que a árvore matriz está inserida. Felippi et al. (2012) afirmam que o índice germinativo de sementes de louro-pardo (Cordia trichotoma) é oscilante, provavelmente em função da variação de cada árvore matriz e seu micro-habitat.

No teste de transmissão foi possível constatar a presença de plântulas sintomáticas apenas no lote RES (4\%), porém ambos os lotes tiveram altos valores de sementes mortas. Essas sementes mortas quando retiradas do substrato encontravam-se em condições de degradação, muito provavelmente em função do ataque de microrganismos (fungos). Como o substrato utilizado passou por um processo inicial de esterilização, esses fungos deviam estar associados internamente ou externamente à semente, e encontraram situações favoráveis para o seu desenvolvimento.

Os gêneros fúngicos identificados no teste de transmissão foram Aspergillus, Penicillium, Fusarium e Alternaria, sendo que, os dois últimos foram constatados associados às plântulas anormais, oriundas do teste de transmissão (Tabela 2). Fusarium sp. apresentou maior incidência numérica, alcançando $17 \%$ para 
o lote RES, entretanto não diferiu da ocorrência no lote SM (14\%). No caso das plântulas anormais, os sintomas observados foram, amarelecimento e murcha do caulículo (Figura 1D), com posterior tombamento da plântula. Santos et al. (2011) identificaram os fungos Fusarium sp., Thielaviopsis paradoxa, Colletotrichum sp., Helminthosporium sp. e Phytophtora palmivora em sementes de pupunha (Bactris gasipaes Kunth. var. gasipaes Henderson), e afirmam que o gênero Fusarium foi transmitido para plântulas; Moers et al. (2012) verificaram Fusarium sp. causando damping-off em plântulas de crambe (Crambe abyssinica Hochst) no estado do Paraná. Isso demonstra que este patógeno é cosmopolita e pouco específico em relação aos hospedeiros, consequentemente de difícil controle.

Os gêneros Penicillium e Aspergillus são considerados patógenos de armazenamento, e tem sua ocorrência aumentada quando as sementes são mantidas em condições inadequadas (Cherobini et al. 2008). Como os testes com sementes de açoita-cavalo foram realizados logo após o beneficiamento, o percentual de associação com esses fungos foi baixo, entre 0 e $3 \%$ (Tabela 2 ).

Figura 1. Qualidade fisiológica e sanitária de sementes de açoita-cavalo. A) Detecção em BDA; B) Detecção em blotter test, com ênfase para estruturas do gênero Fusarium vistas em lupa com aumento de 4x; C) Plântulas normais; D) Plântulas sintomáticas no teste de emergência.

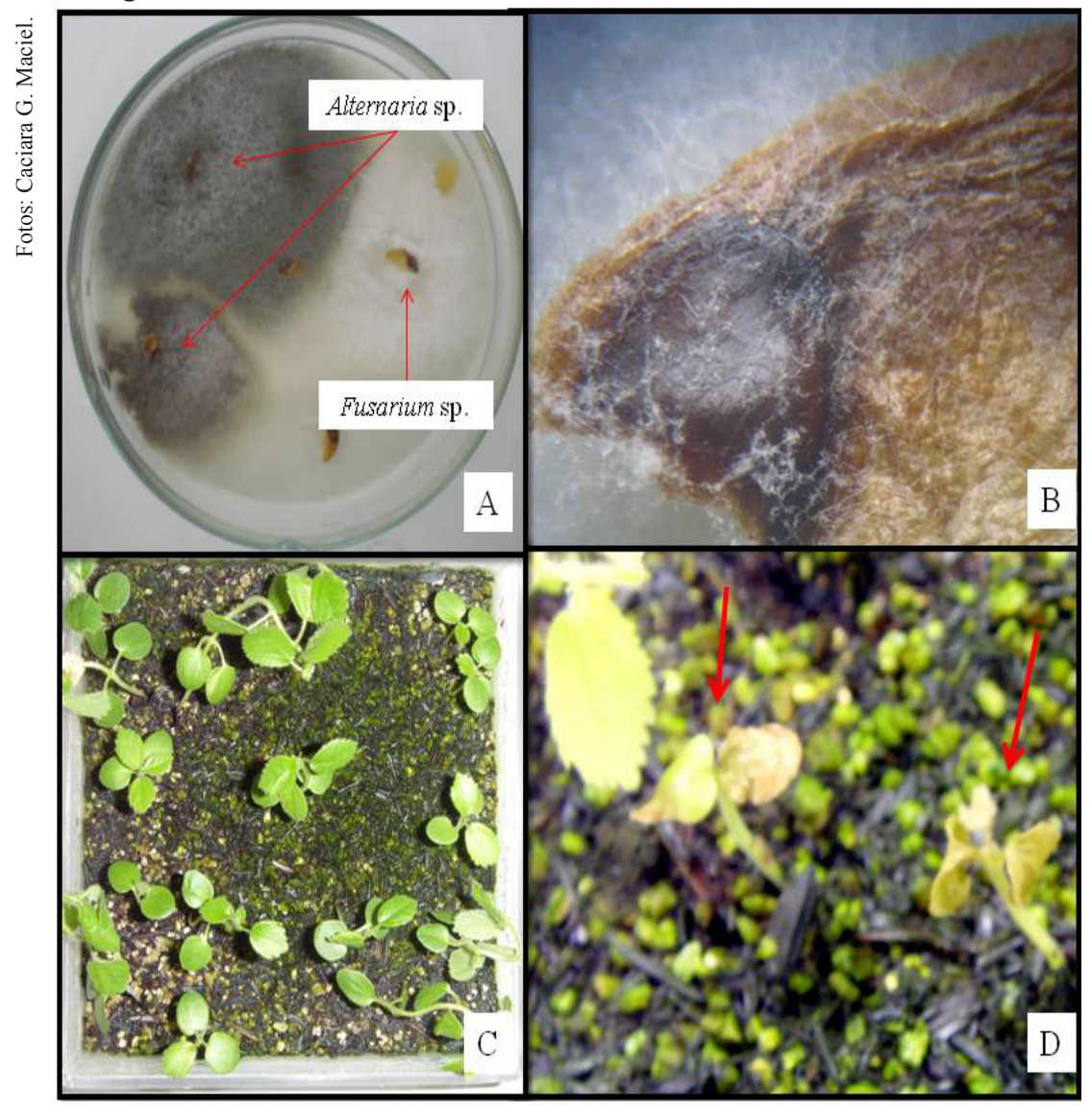

Tabela 2. Incidência fúngica (\%) em plântulas sintomáticas e sementes mortas de açoita-cavalo, obtidas no teste de transmissão, coletadas nos municípios de Santa Maria (SM) e Restinga Seca (RES).

\begin{tabular}{ccccc}
\hline & Fusarium sp. & Alternaria sp. & Aspergillus sp. & Penicillium sp. \\
\hline Lote 1 (SM) & $14 \mathrm{a}^{*}$ & $0 \mathrm{a}$ & $3 \mathrm{a}$ & $2 \mathrm{a}$ \\
Lote $2(\mathrm{RES})$ & $17 \mathrm{a}$ & $3 \mathrm{a}$ & $0 \mathrm{a}$ & $1 \mathrm{a}$ \\
\hline
\end{tabular}

* Médias seguidas pela mesma letra, na coluna, não diferem estatisticamente entre si pelo teste de Tukey ao nível de 5\% de significância. 
Além dos fungos já identificados no teste de transmissão, outros gêneros foram constatados no teste de sanidade. Sendo eles: Rhizoctonia sp., Pestalotiopsis sp., Phoma sp. e Cladosporium sp. Entre os poucos estudos que relatam as condições sanitárias de sementes de açoita-cavalo, Mieth et al. (2007) apontaram o gênero Colletotrichum, além de outros comuns aos do presente trabalho, como, Fusarium, Phoma, Cladosporium e Rhizoctonia.

Independente do método utilizado para detecção (BDA (Figura 1A) ou PF (Figura 1B), o fungo que se destacou foi Fusarium sp., atingindo 44\% no lote SM pelo método PF (Tabela 3). Benetti et al. (2009) também relataram altos valores de Fusarium sp. associado a sementes de cedro (Cedrela fissilis) em ambos os métodos, causando interferência na emergência final de plântulas. Lazarotto et al. (2012) relatam Fusarium sp. e Rhizoctonia sp. como potenciais patogênicos a plântulas de cedro, causando danos nas raízes e posterior tombamento.

Phoma sp. e Cladosporium sp. foram verificados apenas no método PF, em ambos os lotes (Tabela 3). Isso pode estar associado à assepsia realizada na detecção em BDA, que elimina aqueles patógenos superficiais a semente e não permite que sejam identificados. No caso do gênero Pestalotiopsis que foi observado em ambos os lotes, apenas em BDA pode-se dizer que este patógeno estava interno nas estruturas da semente, e só encontrou condições para multiplicação quando aqueles fungos externos foram eliminados pela assepsia.

Tabela 3. Incidência fúngica (\%) em sementes de açoita-cavalo coletadas nos municípios de Santa Maria (SM) e Restinga Seca (RES), pelos testes de sanidade em meio batata-dextrose-ágar (BDA) e papel-filtro (PF).

\begin{tabular}{|c|c|c|c|c|c|c|}
\hline & \multicolumn{6}{|c|}{ Incidência fúngica em meio BDA(\%) } \\
\hline & Rhizoctonia sp. & Fusaruium sp. & Penicillium sp. & Pestalotiopsissp. & Aspergillussp. & Phomasp. \\
\hline Lote $1(\mathrm{SM})$ & $10 \mathrm{~b}$ & $12 \mathrm{a}$ & $6 a$ & $2 \mathrm{a}$ & $0 \mathrm{a}$ & - \\
\hline \multirow[t]{3}{*}{ Lote2(RES) } & $0 \mathrm{a}$ & $20 \mathrm{a}$ & $14 \mathrm{a}$ & $1 \mathrm{a}$ & $20 \mathrm{~b}$ & - \\
\hline & \multicolumn{6}{|c|}{ Incidência fúngica em pape-filtro(\%) } \\
\hline & Alternaria sp. & Fusarium sp. & Penicillium sp. & Cladosporium sp. & Aspergillussp. & Phomasp. \\
\hline Lote $1(\mathrm{SM})$ & $0 \mathrm{a}$ & $44 \mathrm{a}$ & $0 \mathrm{a}$ & $5 a$ & $0 \mathrm{a}$ & $24 \mathrm{a}$ \\
\hline Lote2(RES) & $34 b$ & $41 \mathrm{a}$ & $4 b$ & $2 \mathrm{a}$ & $2 \mathrm{a}$ & $9 \mathrm{a}$ \\
\hline
\end{tabular}

* Médias seguidas pela mesma letra, na coluna, não diferem estatisticamente entre si pelo teste de Tukey ao nível de 5\% de significância.

Observa-se na Tabela 4 que o teste em PF foi capaz de detectar maior variedade de fungos. Isso acontece, pois esse método permite quantificar os fungos externos a semente, diferente do método em BDA, que devido à assepsia superficial realizada, predomina a presença dos fungos internos. Ruiz Filho et al. (2004) compararam PF e BDA e constataram que os dois testes foram eficientes na recuperação dos fungos em sementes de cedro, sendo os seguintes gêneros identificados: Phomopsis, Phoma, Cladosporium, Fusarium, Curvularia, Pestalotia, Penicillium, Aspergillus, Trichoderma, Rhizopus, Chaetomium, Ascochyta e Stilbum. Lazarotto et al. (2010) também verificaram que ambos os métodos de detecção testados (BDA e PF) foram eficientes para a identificação da maioria dos fungos, associados a sementes de paineira (Ceiba speciosa St.-Hill), e destacam aqueles potencialmente patogênicos, tais como Fusarium sp. e Alternaria sp..
A incidência do fungo Pestalotiopsis sp. foi verificada apenas em meio BDA (Tabela 4). Isso indica que esse patógeno estava predominando nas estruturas internas da semente. Por outro lado, Schultz et al. (2003), encontraram Pestalotia sp. em sementes de pau-cigarra (Senna multijuga), independentemente do método utilizado, PF ou BDA.

Através do teste de sanidade com os frutos recémcolhidos de açoita-cavalo (Tabela 5), pode-se dizer que o fungo Fusarium sp. possivelmente foi transmitido do fruto para a semente, semelhante ao relatado por Rego et al. (2009), em estudo realizado com frutos e sementes de capororoca (Myrsine ferruginea). Os autores encontraram os fungos Alternaria sp., Cladosporium sp., Colletotrichum sp., Fusarium sp., Macrophomina sp. e Pestalotia sp. associados aos frutos e sementes de capororoca. De acordo com Menten \& Bueno (1987), 
são diversas as portas de entrada de inóculo externo para infecção das sementes, entre elas: estigma, nectário, pedúnculo da flor ou do fruto e aberturas no fruto. Ainda, segundo esses autores, a cavidade de alguns tipos de frutos pode atuar como câmara úmida para o desenvolvimento de fungos, que é o caso do açoitacavalo.

No caso de Alternaria sp., também foi observada a relação da presença do fungo na semente e no fruto. O patógeno foi apontado no teste de sanidade em PF e
BDA (Tabela 3), na transmissão de fungos via semente (Tabela 2) e associado aos frutos (Tabela 5), e em todos os casos no Lote RES. Alguns autores já evidenciaram a relação entre fungos nos frutos e nas sementes, como por exemplo Rego et al. (2008), que identificaram os gêneros Botryodiplodia, Colletotrichum, Alternaria, Curvularia e Fusarium em sementes e frutos de imbuia (Ocotea porosa Ness. L. Barroso); Ávila et al. (2009) relataram a incidência de Cladosporium sp. e Alternaria sp. em frutos e sementes de pitanga (Eugenia uniflora L.), dentre outros.

Tabela 4. Número de amostras de sementes de açoita-cavalo em que houve ocorrência de fungos em papel-filtro (PF) e batatadextrose-ágar (BDA), número de vezes em que houve coincidência na recuperação de fungos nos dois métodos utilizados e incidência média (\%) de fungos.

\begin{tabular}{lccccc}
\hline & \multicolumn{2}{c}{ Ocorrência $^{\mathbf{1}}$} & \multirow{2}{*}{ Coincidência $^{2}$} & \multicolumn{2}{c}{ Incidência média (\%) $^{\mathbf{3}}$} \\
\cline { 2 - 3 } \cline { 5 - 6 } & PF & BDA & & BDA & PF \\
\hline Rhizoctonia sp. & 0 & 1 & 0 & 5 & 17 \\
Fusarium sp. & 2 & 2 & 2 & 16 & 42,5 \\
Penicillium sp. & 1 & 2 & 1 & 10 & 2 \\
Pestalotiopsis sp. & 0 & 2 & 0 & 1,5 & 0 \\
Aspergillus sp. & 1 & 1 & 1 & 10 & 1 \\
Phoma sp. & 2 & 0 & 0 & 0 & 16,5 \\
Alternaria sp. & 1 & 0 & 0 & 0 & 17 \\
Cladosporium sp. & 2 & 0 & 0 & 0 & 3,5 \\
\hline
\end{tabular}

${ }^{1}$ Número de amostras avaliadas: $2 .{ }^{2}$ Número de vezes em que houve coincidência na recuperação de fungos entre os dois métodos. ( $\sum \%$ de ocorrência nos dois lotes para cada método)/número de lotes).

Tabela 5. Incidência fúngica (\%) em frutos de açoita-cavalo coletados nos municípios de Santa Maria (SM) e Restinga Seca (RES).

\begin{tabular}{cccccc}
\hline Frutos & Fusarium sp. & Penicillium sp. & Aspergillus sp. & Cladosporium sp. & Alternaria sp. \\
\hline Lote 1 (SM) & $35 \mathrm{a}^{*}$ & $0 \mathrm{a}$ & $2,5 \mathrm{a}$ & $2,5 \mathrm{a}$ & $0 \mathrm{a}$ \\
Lote 2 (RES) & $45 \mathrm{a}$ & $2,5 \mathrm{a}$ & $0 \mathrm{a}$ & $0 \mathrm{a}$ & $27,5 \mathrm{~b}$ \\
\hline
\end{tabular}

* Médias seguidas pela mesma letra, na coluna, não diferem estatisticamente entre si pelo teste de Tukey no nível de 5 \% de significância.

\section{Conclusões}

Os métodos de detecção, papel-filtro e BDA, são eficientes e complementares na verificação dos fungos associados a sementes de açoita-cavalo, dessa maneira podem ser utilizados para avaliação sanitária de sementes dessa espécie

Os fungos identificados associados às sementes, independente do método são: Fusarium sp., Alternaria sp., Rhizoctonia sp., Aspergillus sp., Penicillium sp.,
Pestalotiopsis sp., Phoma sp. e Cladosporium sp.

Os fungos presentes nos frutos de açoita-cavalo são: Fusarium sp., Aspergilus sp., Penicillium sp., Cladosporium sp. e Alternaria sp.

Esse trabalho salienta a incidência e transmissão de fungos com potencial patogênico em sementes de açoita-cavalo, e aponta para a necessidade de detecção e controle desses patógenos associados a semente, uma vez que os mesmos podem interferir na qualidade final das mudas. 


\section{Referências}

AVILA, A. L. de; ARGENTA, M. da S.; MUNIZ, M. F. B.; POLETO I.; BLUME E. Maturação fisiológica e coleta de sementes de Eugenia uniflora L. (pitanga), Santa Maria, RS. Ciência Florestal, Santa Maria, v. 19, n. 1, p. 61-68, 2009.

BARNETT, H. L.; HUNTER, B. B. Illustrate genera of imperfect fungi. 3rd ed. Minnesota: Burgess Publishing Company, 1999.

BENETTI, S. C.; SANTOS, A. F. dos; JACCOUD FILHO, D. de S. Levantamento de fungos em sementes de cedro e avaliação da patogenicidade de Fusarium sp. e Pestalotiopsis sp. Pesquisa Florestal Brasileira, Colombo, v. 58, p. 79-83, 2009.

BERNARDI-WENZEL, J.; GARCÍA, A.; CELSO FILHO, J. R.; PRIOLI A. J.; PAMPHILE, J. A. Evaluation of foliar fungal endophyte diversity and colonization of medicinal plant Luehea divaricata (Martius et Zuccarini). Biological Research, Santiago Del Chile, v. 43, p. 375-384, 2010.

BORGES, K. C. de F.; SANTANA, D. G. de; RANAL, M.; DORNELES, M. C.; CARVALHO, M. P. Germinação de sementes e emergência de plântulas de Luehea divaricata Mart. Revista Brasileira de Biociências, Porto Alegre, v. 5, n. 2, p. 1008-1010, 2007.

BOTElHO, L. da S.; MORAES, M. H. D.; MENTEN, J. O. M. Fungos associados às sementes de ipê-amarelo (Tabebuia serratifolia) e ipê-roxo (Tabebuia impetiginosa): incidência, efeito na germinação e transmissão para as plântulas. Summa Phytopathologica, Botucatu, v. 34, n. 4, p. 343-348, 2008.

BRASIL. Ministério da Agricultura, Pecuária e Abastecimento. Regras para análise de sementes. Ministério da Agricultura, Pecuária e Abastecimento. Secretaria de Defesa Agropecuária. Brasília, DF: Mapa/ACS; 2009.

CARVALHO, P. E. R. Açoita-Cavalo (Luehea divaricata). Colombo: Embrapa Florestas, 2008. 9 p. (Embrapa Florestas. Circular técnica, 147).

CHEROBINI, E. A. I.; MUNIZ, M. F. B.; BLUME, E. Avaliação da qualidade de sementes e mudas de cedro. Ciência Florestal, Santa Maria, RS, v. 18, n. 1, p. 65-73, 2008.

FELIPPI M.; MAFFRA C. R. B.; CANTARELLI E. B., ARAÚJO, M. M., LONGH, S. J. Fenologia, morfologia e análise de sementes de Cordia trichotoma (Vell.) Arráb. ex Steud. Ciência Florestal, Santa Maria, RS, v. 22, n. 3, p. 631-641, 2012.

GRAVE, F.; FRANCO E. T. H.; PACHECO, J. P.; SANTOS, S. R. Crescimento de plantas jovens de açoita-cavalo em quatro diferentes substratos. Ciência Florestal, Santa Maria, RS, v. 17, n. 4, p. 289298, 2007.

GRUTKA, T. H. H.; FRIGO, M. S.; FRIGO, E. P.; TESSARO, D. Efeito de diferentes proporções de adubação orgânica sobre o desenvolvimento de mudas de açoita-cavalo (Luehea divaricata). Engenharia Ambiental, Espírito Santo do Pinhal, v. 9, n. 1, p. 17-25, 2012.

LAZAROTTO, M.; MUNIZ, M. F. B.; BELTRAME, R.; SANTOS, Á. F. dos; MACIEL C. G.; LONGHI S. J. Sanidade, transmissão via semente e patogenicidade de fungos em sementes de Cedrela fissilis procedentes da região Sul do Brasil. Ciência Florestal, Santa Maria, RS, v. 22, n. 3, p. 493-503, 2012.
LAZAROTTO, M.; MUNIZ, M. F. B.; SANTOS, Á. F. dos. Detecção, transmissão, patogenicidade e controle químico de fungos em sementes de paineira (Ceiba speciosa). Summa Phytopathologica, Botucatu, v. 36, n. 2, p. 134-139, 2010.

MACIEL, C. G.; MUNIZ, M. F. B.; SANTOS, A. F. dos; LAZAROTTO, M. Detecção, transmissão e patogenicidade de fungos em sementes de Parapiptadenia rigida (angico-vermelho). Summa Phytopathologica, Botucatu, v. 38, n. 4, p. 323-328, 2012.

MAGUIRE, J. D. Speed of germination-aid in selection and evaluation for seedling emergence and vigours. Crop Science, Madison, v. 2, n. 1, p. 176-177, 1962.

MENTEN, J. O. N.; BUENO, J. T. Transmissão de patógenospelas sementes. In: SOAVE, J.; WETZEL, M. M. V. S. Patologia de Sementes. Campinas: Cargill, 1987. p. 164-191.

MIETH, A. T.; PIVETA, G.; PACHECO, C.; HAMANN, F. A.; RODRIGUES, J.; MUNIZ, M. F. B. Influência de extrato vegetal na qualidade sanitária e fisiológica em sementes de Luehea divaricata (Açoita-cavalo). Revista Brasileira de Agroecologia, Curitiba, v. 2, n. 2, p. 1240-1242, 2007.

MOERS, E. M.; KUHN, O. J.; GONÇALVES J. R. A. C.; FRANZENER, G.; STANGARLIN, J. R. Levantamento de doenças na cultura do crambe (Crambe abyssinica Hochst) na região oeste do Paraná. Scientia Agraria Paranaensis, Cascavel, v. 11, n. 1, p 35-48, 2012.

PIÑA-RODRIGUES, F. C. M. Guia prático para a colheita e manejo de sementes florestais tropicais. Rio de Janeiro: Idaco, 2002. 40 p.

REGO, S. S.; SANTOS, A. F. dos; MEDEIROS, A. C. de S. Fungos associados aos frutos e sementes de capororoca (Myrsine ferruginea) Myrsinaceae. Pesquisa Florestal Brasileira, Colombo, n. 58, p. 85-88, 2009.

REGO, S. S.; SANTOS, Á. F. dos; MEDEIROS, A. C. de S.; JACCOUD FILHO, D. de S. Fungos associados a frutos e sementes de imbuia (Ocotea porosa Ness. L. Barroso). Summa Phytopathologica, Botucatu, v. 34, n. 4, p. 378-378, 2008.

RUIZ FILHO, R. R.; SANTOS, A. F. dos; MEDEIROS, A. C. S.; JACCOUD FILHO, D. S. Fungos associados às sementes de cedro. Summa Phytopathologica, Botucatu, v. 30, n. 4, p. 494-496, 2004.

SARMENTO, M. B.; VILLELA, F. A. Sementes de espécies florestais nativas do Sul do Brasil. Revista Brasileira de Sementes, Brasília, DF, v. 20, n. 1/2, p. 39-44, 2010.

SANTOS, A. F. dos; MACIEL, C. G.; FOWLER, J. A. P. Detecção de fitopatógenos em sementes de pupunheira e transmissão de Fusarium sp. para plântulas. Colombo: EMBRAPA-CNPF, 2011. 3 p. (Embrapa Florestas. Comunicado técnico, 277).

SCHULTZ, V. S.; SANTOS, A. F. dos; MEDEIROS A. C. S. Qualidade sanitária de sementes de pau-cigarra (Senna multijuga). Boletim de Pesquisa Florestas, Colombo, n. 47, p. 123-128, 2003.

SILVA, L. G. da; COSMI, F. C.; JESUS JUNIOR, W. C. de; SOUZA, A. F. de; MORAES W. B. Efeito do tratamento químico na sanidade de sementes de espécies florestais. Ciência Florestal, Santa Maria, RS, v. 21, n. 3, p. 469-474, 2011.

Pesq. flor. bras., Colombo, v. 33, n. 75, p. 331-338, jul./set. 2013 
SILVA, L. C. da. Avaliação do potencial para armazenamento de sementes de Açoita-cavalo (Luehea divaricata Mart. - Tiliaceae). 2004. 28 f. Dissertação (Mestrado em Ciências) - Universidade Federal de Pelotas, Pelotas.
SOUZA, A. de A.; NASCIMENTO, C. R. do; SILVA, A. da C. D. da; BARBOSA, R. N. T.; ANDRADE, J. K. C. de; NASCIMENTO, J. F.do. Incidência de fungos associados a sementes de ipê-rosa (Tabebuia impetiginosa) e ipê-amarelo (Tabebuia ochracea) em Roraima. Revista Agro@mbiente On-line, Roraima, v. 6, n. 1, p. 34-39, 2012. 